INRA Prod. Anim., 1996, 9 (1), 9-23
B. MALPAUX, C. VIGUIÉ, J.C. THIÉRY,
P. CHEMINEAU

INRA Station de Physiologie de la Reproduction des Mammifères Domestiques

37380 Nouzilly

\section{Contrôle photopériodique de la reproduction}

\author{
La durée du jour est responsable du saisonnement de la reproduction \\ dans de nombreuses espèces. Le cheminement du signal \\ photopériodique de l'œil aux gonades fait intervenir des mécanismes \\ variés, nerveux et endocriniens.
}

L'existence de saisons, avec les variations associées des facteurs climatiques, est l'un des principaux défis auxquels les organismes vivants sont confrontés dans les zones tempérées. Différents processus physiologiques existent qui permettent de moduler les fonctions physiologiques de l'organisme en fonction des saisons. Parmi ceux-ci, on peut citer, selon les espèces, l'hibernation, les variations de croissance du poil et la mue, la mise en réserve d'énergie dans le tissu adipeux brun, la migration... (Gwinner 1986). Toutefois, un processus commun à la majorité des espèces animales est la mise en sommeil de la fonction de reproduction quand une fécondation entraînerait des naissances à un moment défavorable à la survie des jeunes. Cette sai-

\section{Résumé}

Les variations annuelles de la durée du jour, ou photopériode, sont responsables de l'alternance entre une saison sexuelle et une saison de repos sexuel dans la plupart des espèces animales. Selon sa durée, la photopériode peut exercer une action stimulante ou inhibitrice sur l'activité de reproduction. Toutefois, en l'absence d'information photopériodique, l'animal exprime un rythme endogène de reproduction et le rôle principal de la photopériode dans les conditions naturelles semble être de synchroniser ce rythme interne des animaux.

L'information lumineuse est perçue par la rétine et, après un trajet nerveux, elle est transformée en un signal endocrinien, le rythme de sécrétion de mélatonine. Cette hormone est sécrétée uniquement pendant la nuit. La mélatonine agit au niveau du système nerveux central pour modifier la sécrétion pulsatile de LHRH. Son action sur les neurones à LHRH n'est pas directe mais met en jeu des interneurones. Par ailleurs, la modification de sécrétion de LHRH est observable avec un délai important par rapport au changement du rythme de sécrétion de mélatonine, de 40 à 60 jours chez la brebis. Les modifications de sécrétion de LHRH induisent à leur tour des changements de sécrétion des gonadotropines et en conséquence des variations d'activité des gonades. sonnalité de la reproduction aboutit généralement à des naissances se déroulant en fin d'hiver ou au printemps ce qui fournit les conditions les plus favorables de développement aux jeunes avant l'hiver suivant. Pour les espèces dont la gestation dure quelques semaines (rongeurs, incubation pour les oiseaux) ou environ un an (équin), la période d'activité sexuelle se déroule au printemps. A l'opposé, pour des espèces à durée de gestation de l'ordre de 6 mois (ovins, caprins, cervidés...), la période d'activité sexuelle se déroule pendant l'automne pour donner des naissances au printemps suivant (Ortavant et al 1985, Martinet et Mondain-Monval 1991). Chez certaines espèces (vison, chevreuil...), la saison influence non seulement la production de gamètes par les gonades mais également la période d'implantation ; ainsi, chez le chevreuil, après des fécondations pouvant se produire en juillet-août, l'implantation a lieu en décembre-janvier pour une période de naissance en mai-juin (Thimonier et Sempéré 1989).

La nécessité de prévoir quelques mois à l'avance le moment favorable aux naissances implique l'utilisation par l'animal d'un indicateur fiable du moment de l'année. Le facteur de l'environnement utilisé par la majorité des espèces est la variation de la durée journalière d'éclairement, ou photopériode. Cet article analyse d'abord le rôle de la photopériode et la manière dont celle-ci contrôle le cycle annuel de reproduction, puis présente les mécanismes par lesquels l'information lumineuse module la fonction de reproduction. Cette revue sera limitée aux mammifères et la plupart des faits expérimentaux rapportés concerneront les espèces ovine ou caprine. Il sera fait appel à d'autres espèces pour des questions non résolues chez ces deux espèces. 


\section{1 / Contrôle du cycle annuel de reproduction}

\section{1 / Variations saisonnières d'activité sexuelle et de sécrétion d'hormones gonadotropes}

Chez les ovins, sous nos latitudes, la reproduction a un caractère saisonnier marqué, caractérisé par l'alternance d'une période de repos sexuel au printemps et en été, et d'une période d'activité sexuelle en automne et en hiver (Yeates 1949, Thimonier et Mauléon 1969, Ortavant et al 1985). La période de repos sexuel est caractérisée chez la brebis par l'établissement d'un état d'anœstrus, le plus souvent associé à l'absence d'ovulation (figure 1). En revanche, la saison sexuelle se caractérise par la succession de cycles œstriens tous les 15 à 18 jours (Thimonier et Mauléon 1969). Chez le bélier, la production

Figure 1. Variations d'activité ovulatoire chez des brebis lle-de-France en photopériode naturelle ou soumises à une alternance entre 3 mois de jours longs et 3 mois de jours courts. Les durées du jour et de la nuit sont représentées en haut de chaque graphique par des surfaces respectivement claires et foncées (adapté de Thimonier 1989).

Dans les espèces à reproduction saisonnière, on peut décaler les phases de repos et d'activité sexuels en appliquant un régime lumineux alternant jours longs et jours courts.

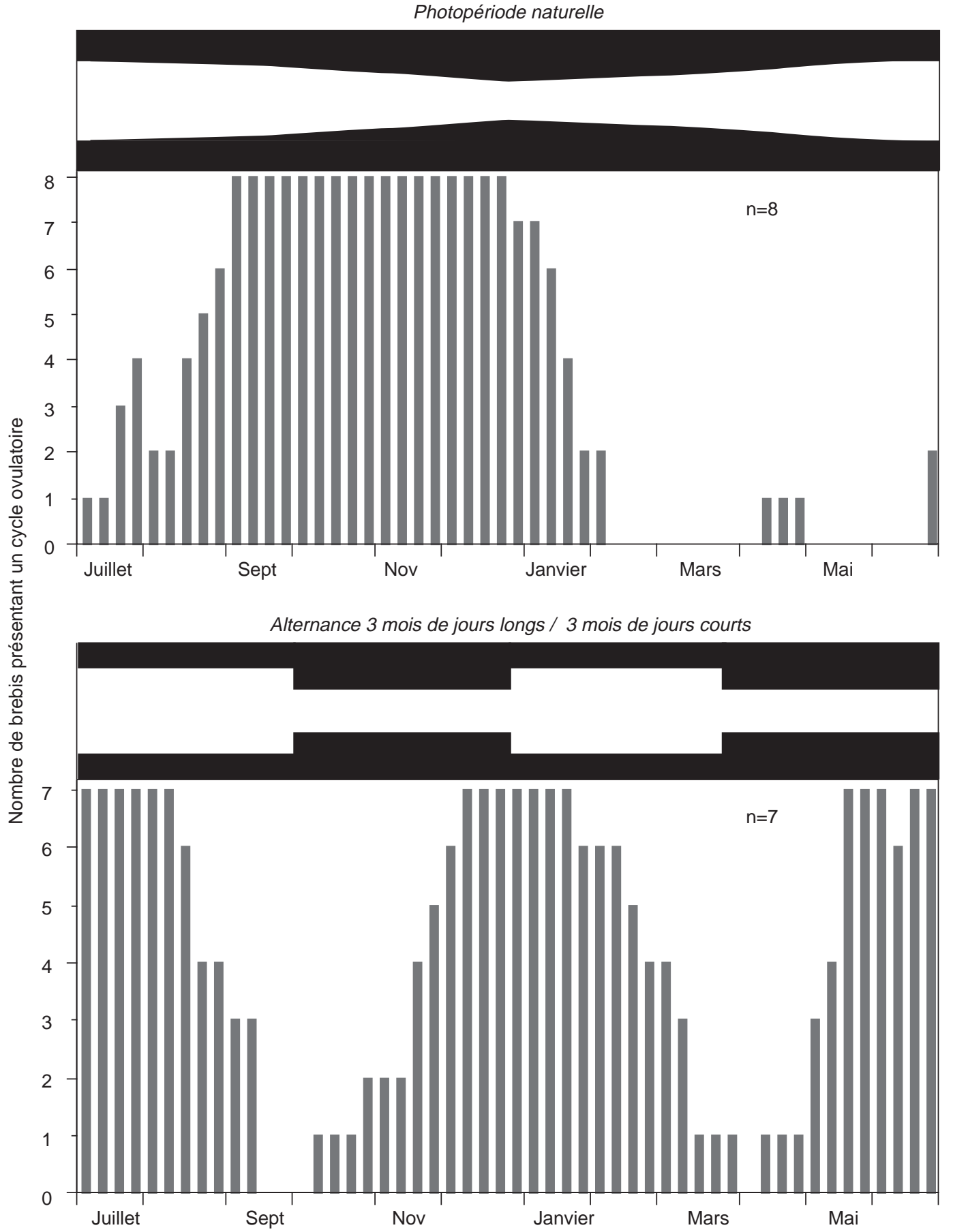


spermatique varie également au cours de l'année. Ainsi, chez le bélier Ile-de-France, la production quotidienne de spermatozoïdes est quatre fois plus élevée en automne qu'au printemps (Dacheux et al 1981).

Les variations d'activité sexuelle résultent de changements de sécrétion des hormones gonadotropes, LH (hormone lutéinisante) et FSH (hormone folliculo-stimulante) (Karsch et al 1984). La saison influence la fréquence des épisodes de libération de LH (sécrétion pulsatile) qui est la caractéristique la plus importante de la sécrétion de cette hormone, par deux mécanismes complémentaires : l'un est dépendant des stéroïdes gonadiques, l'autre est indépendant de ceux-ci (Pelletier et Ortavant 1975). Chez la brebis ou le bélier castrés, la sécrétion pulsatile de LH est plus faible pendant la saison de repos sexuel que pendant la saison sexuelle (1 vs 2 pulses par heure chez la brebis castrée ; Pelletier et Ortavant 1975, Montgomery et al 1985, Robinson et al 1985a). Cette différence de sécrétion de LH entre saisons de repos et d'activité sexuels est très fortement accrue en présence d'œstradiol ou de testostérone (Pelletier et Ortavant 1975, Karsch et al 1984, Chemineau et al 1988). Ainsi, chez la brebis ovariectomisée traitée avec un implant d'œstradiol délivrant des taux analogues à ceux observés en milieu de phase folliculaire, on observe 1 pulse toutes les 12 à 24 heures pendant la saison d'anœstrus contre 1 pulse toutes les 30 minutes pendant la saison sexuelle (Karsch et al 1984). Par conséquent, les changements de sensibilité à l'œstradiol chez la femelle et à la testostérone chez le mâle sont le principal mécanisme responsable de la saisonnalité de la reproduction. Ces variations de sensibilité à l'œstradiol sont à l'origine d'un modèle expérimental très largement utilisé : la brebis ovariectomisée et traitée avec un implant sous-cutané délivrant une quantité constante d'œstradiol. Les concentrations plasmatiques de LH qui sont mesurées chez cet animal reflètent les modifications de sensibilité à l'œstradiol et sont parfaitement corrélées aux variations d'activité ovulatoire chez la femelle entière (Karsch et al 1984).

\section{2 / Contrôle du cycle annuel de reproduction par la photopériode}

Le rôle de la photopériode a été mis en évidence dans une série d'expériences montrant que la période d'activité sexuelle peut être déplacée dans le temps en modifiant le régime photopériodique sans changer les autres facteurs de l'environnement. Par exemple, l'inversion du cycle photopériodique annuel cause un décalage de 6 mois de la saison sexuelle et la réduction à 6 mois du cycle photopériodique provoque l'apparition de deux saisons sexuelles par an (Mauléon et Rougeot 1962, Thwaites 1965).

Par ailleurs, l'utilisation d'alternance entre des jours courts et des jours longs constants montre que les passages en jours courts et en jours longs sont respectivement suivis d'une stimulation et d'une inhibition de l'activité de reproduction, avec cependant un temps de latence dans chaque cas (figure 1). Par exemple, chez des brebis soumises de manière alternée à des jours courts et des jours longs (90 jours de traitement pour chaque photopériode), le déclenchement de l'activité ovulatoire ou l'augmentation de la sécrétion de $\mathrm{LH}$ se produit 40 à 60 jours après le passage jours longs/jours courts alors que les évolutions inverses se produisent 20 à 30 jours après le passage jours courts/jours longs (Karsch et al 1984, Thimonier 1989)

Les effets stimulants des jours courts ainsi que le déclenchement de la saison sexuelle pendant les jours décroissants de fin d'été ou d'automne ont conduit à qualifier les ovins d'animaux de "jours courts ". De plus, le déclenchement de la saison sexuelle, qui se produit dans la plupart des races en août-septembre, semblait résulter de l'action stimulante des jours décroissants perçus après le solstice d'été. A l'opposé, l'arrêt de cette activité entre janvier et avril semblait être provoqué par l'augmentation de la durée du jour après le solstice d'hiver. Il apparaît maintenant que la régulation du cycle annuel de reproduction est beaucoup plus compliquée, avec en particulier la survenue d'états photoréfractaires. En effet, chez la brebis, la diminution de la durée du jour après le solstice d'été ne semble pas être responsable du déclenchement de la saison sexuelle. L'activité sexuelle de brebis maintenues sous une durée du jour constante équivalente à celle du solstice d'été à partir de celui-ci, débute au même moment que chez des animaux témoins maintenus en photopériode naturelle (Thimonier et al 1978, Robinson et al 1985b, Worthy et al 1985). Il en est de même pour des brebis maintenues sous une photopériode continuellement croissante à partir de l'équinoxe de printemps et même après le solstice d'été (Malpaux et al 1989). Au moment du déclenchement de la saison sexuelle, les animaux semblent donc être réfractaires à l'action inhibitrice des jours longs ambiants. En ce qui concerne la fin de la saison sexuelle, la même conclusion s'applique. Les brebis deviennent réfractaires aux effets stimulants des jours courts ambiants. Des brebis maintenues en jours courts à partir du solstice d'hiver ou exposées à une photopériode continuellement décroissante à partir de l'équinoxe d'automne cessent leur activité sexuelle ou gonadotrope au même moment que les témoins (Worthy et Haresign 1983, Robinson et Karsch 1984, Malpaux et al 1988c).

Ces états réfractaires critiques au déroulement normal de la saison sexuelle pourraient être l'expression d'un rythme endogène de reproduction. L'existence d'un tel rythme a été démontrée chez les ovins comme dans de nombreuses autres espèces : des animaux maintenus en jours courts ou longs constants pendant plusieurs années continuent à montrer des alternances entre périodes de repos

\section{Chez des animaux maintenus sous photopériode constante pendant plusieurs années, un rythme de reproduction persiste, mais il n'est plus synchrone entre animaux.}


et d'activité sexuelles (Ducker et al 1973, Howles et al 1982, Gwinner 1986, Karsch et al 1989, Thimonier 1989). Toutefois, ces périodes d'activité deviennent désynchronisées entre animaux et par rapport à la saison sexuelle normale. La période de ce cycle endogène varie généralement entre 8 et 10 mois. Par exemple, des brebis Suffolk exposées à des jours courts constants pendant 4 ans, montrent des variations d'activité gonadotrope. Ces cycles de sécrétion de LH ne sont pas synchronisés entre animaux et sont caractérisés par une période différente de 1 an (Karsch et al 1989). Le rôle de la photopériode dans les conditions naturelles pourrait donc être de synchroniser ce rythme endogène de reproduction pour lui imposer une période égale à un an.

Il est important de noter que la perception de la photopériode durant certaines périodes critiques de l'année pourrait suffire à entraîner le rythme endogène de reproduction. Ainsi, chez la brebis, les résultats de diverses expériences (Malpaux et al 1989, Malpaux et Karsch 1990, Wayne et al 1990, Woodfill et al 1991) suggèrent que les jours longs de printemps jouent un rôle central pour entraîner le rythme endogène de reproduction et, en particulier, déterminer le moment de déclenchement de la saison sexuelle en fin d'été. Les jours courts interviendraient ensuite pour maintenir cette activité (figure 2 ). Ce modèle a été proposé à la suite de résultats expérimentaux obtenus chez la brebis et sa validité

Figure 2. Modèle pour la régulation photopériodique du cycle annuel de reproduction chez la brebis. Le cycle annuel de reproduction est l'expression d'un rythme endogène de reproduction et le rôle de la photopériode est de synchroniser ce rythme. Les durées du jour croissantes de printemps synchronisent un processus interne qui se termine par un déclenchement obligatoire de la saison sexuelle. Les jours longs jouent également un rôle inhibiteur pour inhiber la fonction sexuelle au moment du solstice d'été. Après le début de la saison sexuelle, les jours courts exercent un effet stimulant qui permet de prolonger l'activité sexuelle jusqu'à son arrêt obligatoire après le solstice d'hiver (adapté de Malpaux et al 1989).

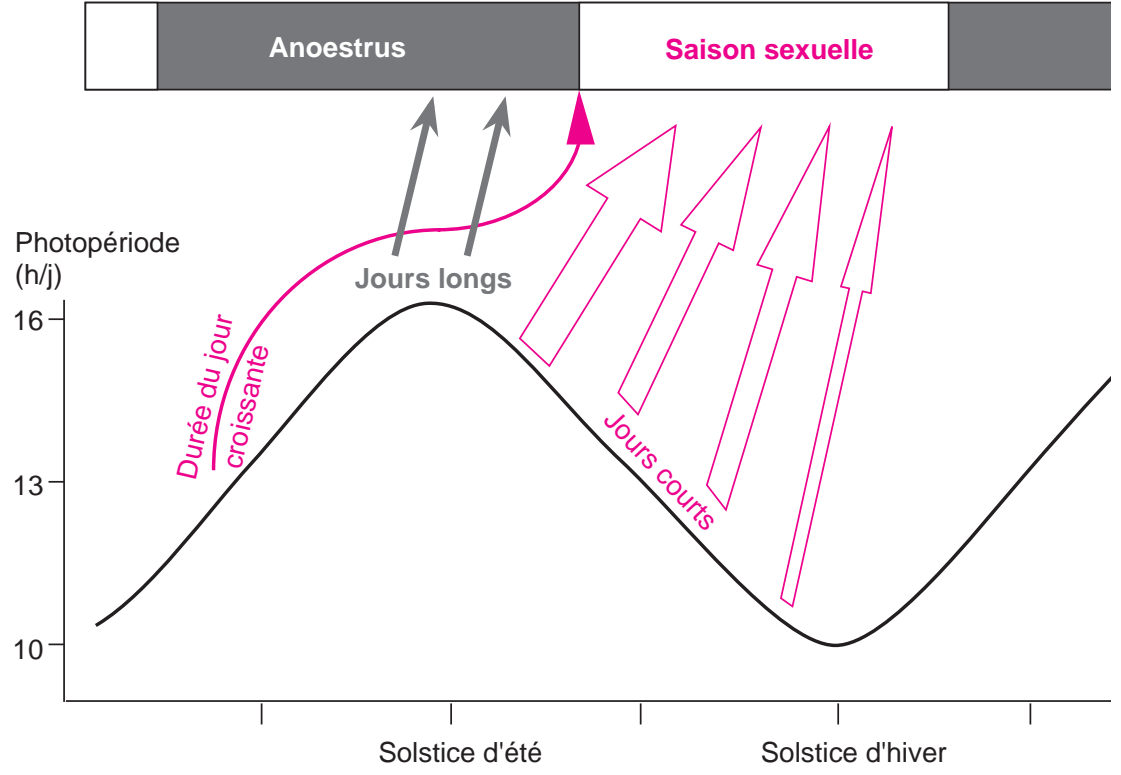

mériterait d'être mise à l'épreuve chez les béliers et dans d'autres espèces. Mais la principale conclusion est que la notion d'animal de « jours courts » ou de «jours longs » doit être utilisée avec prudence. En effet, si ces termes conservent leur valeur d'un point de vue descriptif, ils peuvent conduire à des imprécisions sur le plan mécanistique : le déclenchement de la saison sexuelle est synchronisé par les jours longs de printemps chez un animal dit de "jours courts » comme la brebis.

\section{2 / Mécanismes d'action de la photopériode}

\section{1 / Traduction de l'information photopériodique en un message hormonal}

Chez les mammifères, l'information photopériodique est perçue par la rétine et transmise par voie nerveuse à la glande pinéale en plusieurs étapes. L'information photopériodique est transmise de la rétine aux noyaux suprachiasmatiques par l'intermédiaire de la voie monosynaptique rétino-hypothalamique (figure $3 \mathrm{a}$; Herbert et al 1978, Legan et Winans 1981). A partir de cette structure hypothalamique, le signal est transporté au noyau hypothalamique paraventriculaire (figure $3 a$ et $b$ ), puis dans une colonne de cellules intermédiolatérales située dans la moelle thoracique et ensuite aux ganglions cervicaux supérieurs (figure $3 \mathrm{a}$; Lincoln 1979, Swanson et Kuypers 1980, Klein et al 1983). Le signal parvient enfin à la glande pinéale par les neurones sympathiques postganglionaires. L'importance fonctionnelle de ces voies photoneuroendocriniennes a été démontrée expérimentalement chez le rat et il est admis qu'elles sont similaires chez les autres mammifères. Chez le mouton, l'existence d'une voie rétino-hypothalamique a été démontrée (Legan et Winans 1981). Par ailleurs, dans cette espèce, le rôle de la rétine, des ganglions cervicaux supérieurs et des noyaux suprachiasmatiques a été établi en montrant que la lésion de ces structures modifiait la réponse à la photopériode (Lincoln 1979, Domanski et al 1980, Legan et Karsch 1983, Tessonneaud et al 1995). Ainsi, des béliers de race Soay soumis à une alternance de périodes de 16 semaines de jours longs et de 16 semaines de jours courts expriment des cycles d'activité sexuelle (variations de poids testiculaire) qui sont synchronisés entre animaux et dont la période est proche de 32 semaines (Lincoln 1979). Après ganglionectomie cervicale supérieure, le poids testiculaire de ces animaux ne varie plus de manière significative. De même, des brebis de race Suffolk exposées à une alternance de 90 jours courts et 90 jours longs se caractérisent par un cycle très marqué de sécrétion de LH. Après pinéalectomie les cycles de sécrétion de 
Figure 3. Coupe sagittale de cerveau (a) et d'hypothalamus (b) de mouton.

a. L'information lumineuse chemine de l'œil à la glande pinéale en passant par les noyaux suprachiasmatiques (NSC), les noyaux hypothalamiques paraventriculaires et les ganlions cervicaux supérieurs (EM : éminence médiane). La fenêtre grisée constitue le cadre de la partie b de la figure.

b. Ce schéma illustre les sites potentiels d'action de la mélatonine dans l'hypothalamus de la brebis. Chaque sphère représente le site d'implantation de mélatonine chez un animal et sa taille est indicative de l'étendue de la diffusion de la mélatonine. Les sphères rouges et blanches indiquent respectivement les sites où la mélatonine est active ou inactive pour modifier l'activité de l'axe gonadotrope. Les lignes grises représentent les contours du troisième ventricule (adapté de Malpaux et al 1993).

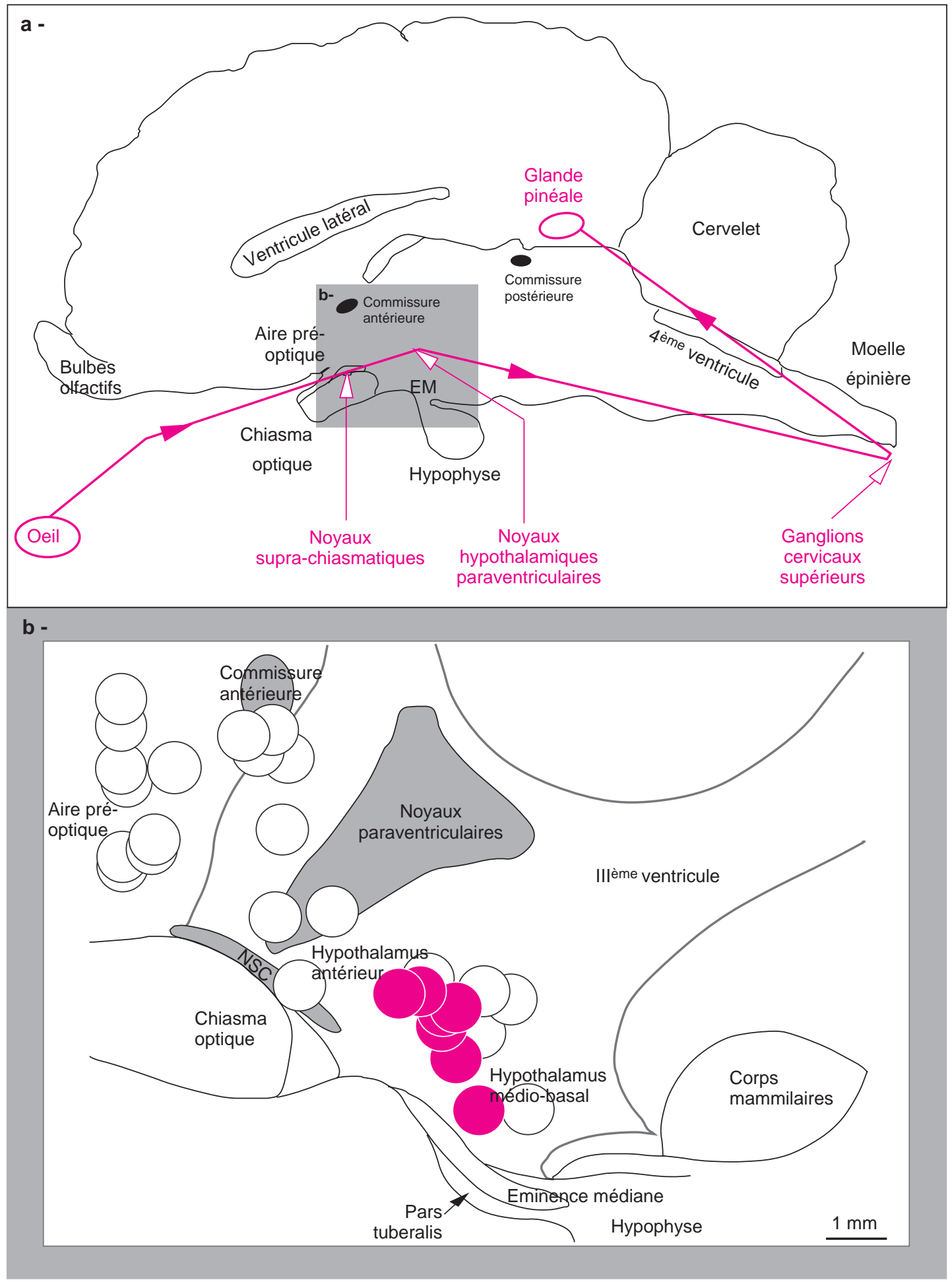

\section{Chez les mammifères, l'information photopériodique est perçue par la rétine et transmise par voie nerveuse à la glande pinéale qui sécrète la mélatonine. Celle-ci agirait sur} l'hypothalamus médio-basal. 
LH persistent, mais les périodes de sécrétion élevée se produisent à des moments variables selon les animaux ; de plus, la période de ces cycles est très différente de celle du régime photopériodique (Bittman et al 1983b).

La glande pinéale n'émet pas de projections nerveuses, son influence sur les fonctions physiologiques met donc en jeu un facteur endocrinien. La principale hormone sécrétée par la glande pinéale est la mélatonine et c'est elle qui traduit les effets de la photopériode sur la fonction de reproduction.

\section{2 / La mélatonine}

\section{a / Rythme de sécrétion}

La mélatonine est sécrétée par la glande pinéale selon un rythme jour/nuit bien défini (Rollag et Niswender 1976, Arendt 1986). Chez les ovins et les caprins, les taux plasmatiques diurnes sont faibles, le plus souvent non détectables avec les dosages radioimmunologiques disponibles $(<5 \mathrm{pg} / \mathrm{ml})$, alors que les taux nocturnes sont élevés et varient de 100 à $500 \mathrm{pg} / \mathrm{ml}$ pour les ovins et de 50 à $150 \mathrm{pg} / \mathrm{ml}$ pour les caprins (Malpaux et al 1987, Delgadillo et Chemineau 1992, figure 4). Ce rythme nycthéméral de sécrétion de mélatonine est un rythme endogène. En effet, lorsque des animaux sont maintenus en obscurité constante, la sécrétion de mélatonine demeure rythmique mais la période du cycle est différente de 24

Figure 4. Profils nycthéméraux de sécrétion de mélatonine chez des boucs alpins $(n=6)$ en jours courts (8 heures d'éclairement par jour) et en jours longs (16 heures d'éclairement par jour). La période obscure est représentée en noir (adapté de Delgadillo et Chemineau 1992).
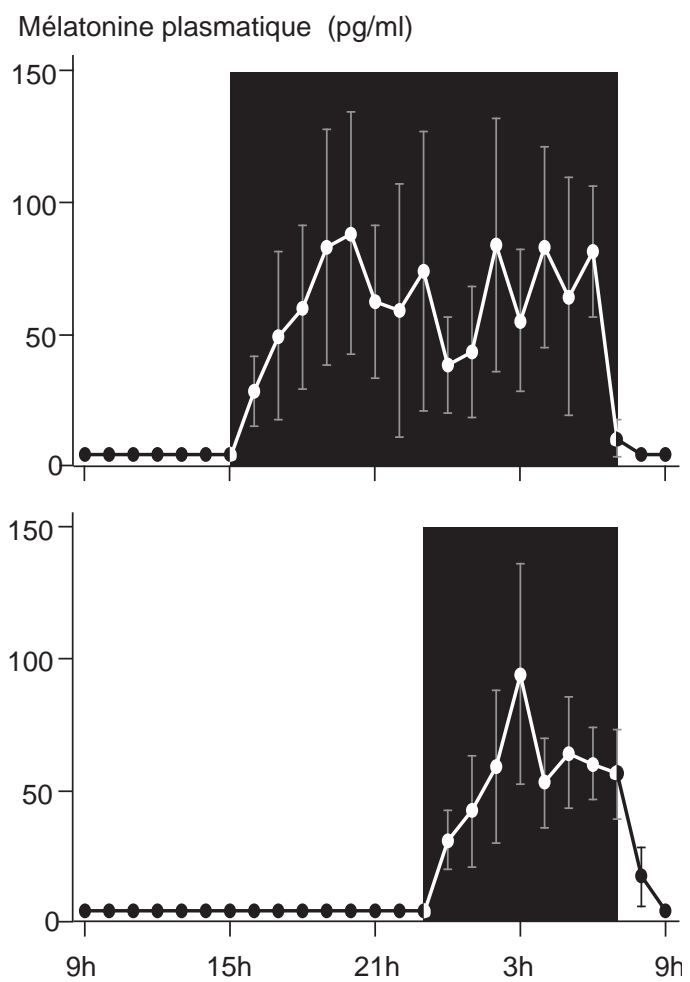

heures et celui-ci n'est pas synchronisé entre animaux (Ebling et al 1988). Le rôle de la lumière est donc de synchroniser ce rythme endogène sur une période de 24 heures. Cet effet implique l'horloge circadienne de l'organisme, les noyaux suprachiasmatiques, qui contrôle la sécrétion de mélatonine. Il est à noter que la lumière exerce également un effet inhibiteur de la sécrétion de mélatonine. En effet, l'illumination des animaux en cours de nuit provoque une chute des niveaux plasmatiques de mélatonine.

La sécrétion exclusivement nocturne de la mélatonine est observée chez tous les mammifères avec toutefois des différences de niveaux nocturnes importants entre espèces $(20$ à 30 $\mathrm{pg} / \mathrm{ml}$ chez le porc domestique, 50 à $100 \mathrm{pg} / \mathrm{ml}$ chez l'homme, quelques centaines de $\mathrm{pg} / \mathrm{ml}$ chez les ovins) (Broadway et al 1987, Paterson et Foldes 1994). Une autre différence interspécifique concerne la forme du profil de sécrétion. Chez les ovins et les caprins, la sécrétion débute très rapidement après le début de la nuit (moins de 10 minutes) et ensuite les niveaux demeurent élevés pendant le reste de la nuit (Malpaux et al 1988c). Au cours de la nuit, les niveaux de mélatonine varient considérablement, ce qui suggère une libération épisodique de cette hormone (Malpaux et al 1987, 1988a). Chez d'autres espèces, en particulier le hamster doré et l'homme, le déclenchement de la sécrétion est plus lent pour atteindre une sécrétion maximale seulement vers le milieu de la nuit (Hoffman 1981, Broadway et al 1987). Chez les ovins, bien que les niveaux de mélatonine varient beaucoup au cours de la nuit et également entre animaux, le niveau nocturne moyen de mélatonine est une caractéristique très répétable des animaux (coefficient de répétabilité de 0,7 chez des brebis Ile-deFrance ; Beltran de Heredia et al 1993).

La mélatonine parvient dans la circulation sanguine par l'intermédiaire de la veine de Galien qui draine la glande pinéale. La mélatonine est également présente dans le liquide céphalo-rachidien à des concentrations 2 à 10 fois plus élevées que dans la circulation périphérique (Kanematsu et al 1989, Shaw et al 1989). Etant donné le faible volume de liquide céphalo-rachidien, la quantité de mélatonine ainsi libérée dans ce dernier est faible, $0,1 \%$ de celle libérée dans le sang. L'importance fonctionnelle de ces taux élevés reste toutefois inconnue.

Bien que la mélatonine soit synthétisée dans d'autres structures que la glande pinéale (rétine, glande de Harder...), la pinéalectomie conduit à des taux nocturnes de mélatonine non détectables, ce qui indique que la glande pinéale est la source principale de cette hormone.

\section{b / Synthèse et dégradation de la mélatonine}

La mélatonine est synthétisée à partir du tryptophane dans les pinéalocytes. Contrairement à la plupart des autres hormones, la 
mélatonine n'est pas stockée dans des vésicules avant sa libération (Reiter 1981). La voie de synthèse inclut successivement le 5-hydroxytryptophane, la sérotonine, la N-acétylsérotonine et enfin la mélatonine (figure 5 ; Sugden 1989). La transformation du tryptophane en 5-hydroxytryptophane par la tryptophane hydroxylase est une étape limitante de la synthèse de sérotonine dans la glande pinéale, mais la régulation circadienne de la sécrétion de mélatonine semble se faire par l'intermédiaire de la $\mathrm{N}$-acétyl-transférase

Figure 5. Voie de synthèse de la mélatonine dans la glande pinéale (adapté de Martinet et MondainMonval 1991).

Tryptophane<smiles>NC(=O)Cc1c[nH]c2ccccc12</smiles>
$\mathrm{H}$

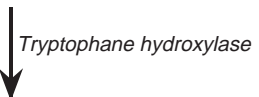

5-hydroxytryptophane<smiles>NC(Cc1c[nH]c2ccc(O)cc12)C(=O)O</smiles>
Aminoacide aromatique décarboxylase

Sérotonine

(5-hydroxytryptamine)

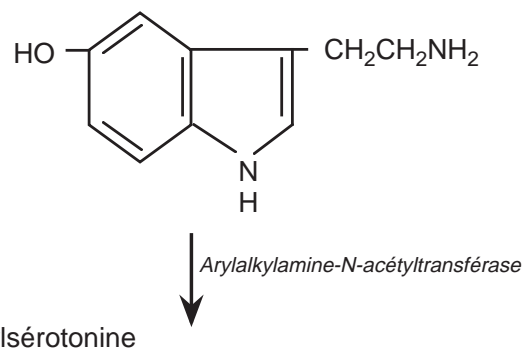

$\mathrm{N}$-acétylsérotonine

(N-acétyl-5-hydroxytryptamine)<smiles>CC(=O)NCCc1c[nH]c2ccc(O)cc12</smiles>

Mélatonine

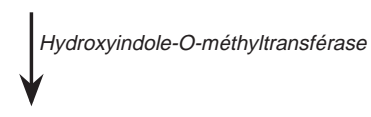

(N-acétyl-5 méthoxytryptamine)<smiles>COc1ccc2[nH]cc(CCNC=O)c2c1</smiles>

(NAT) qui catalyse la transformation de la sérotonine en $\mathrm{N}$-acétyl-sérotonine.

Le début de la nuit est suivi par une augmentation rapide de la libération de noradrénaline au niveau de la glande pinéale par les terminaisons des neurones provenant des ganglions cervicaux supérieurs. La noradrénaline se lie à la fois à des récepteurs adrénergiques $\alpha 1$ et $\beta$ ce qui provoque une augmentation d'AMPc (Klein 1985). La participation exacte de ces deux types de récepteurs reste à établir. Ils pourraient agir en synergie ou la stimulation des récepteurs $\alpha 1$ pourrait potentialiser l'activation des récepteurs $\beta$ (Klein 1985). L'AMPc active une protéine kinase qui, à son tour, stimule l'activité de la NAT. Chez le mouton, des travaux récents suggèrent l'existence d'un mécanisme de régulation de la sécrétion de mélatonine indépendant de la NAT et qui serait dépendant du calcium (Van Camp et al 1991).

Chez les mammifères, la mélatonine est métabolisée en 6-hydroxy-mélatonine par le foie et les reins (Yu et al 1993). Ce métabolite est excrété dans l'urine sous forme sulphatée ou glucuronée. La mélatonine est également métabolisée dans le cerveau en N-acétyl-5méthoxykénurénamine (Hirata et al 1974).

\section{c / Importance de la sécrétion de mélatonine}

L'importance de la mélatonine dans le contrôle de la reproduction a été démontrée par la possibilité de produire des effets jours courts chez des animaux exposés à des jours longs. En jours longs, la mélatonine est sécrétée pendant une durée courte, par exemple 8 heures en 16L (16 heures de lumière et 8 heures d'obscurité). Plusieurs auteurs ont montré qu'il est possible d'administrer de la mélatonine de manière continue (implants sous-cutanés) ou en milieu de journée (injection ou incorporation à la nourriture) pour augmenter la durée de présence de taux élevés de mélatonine. Ces traitements causent une stimulation de l'activité de reproduction chez des brebis ou des chèvres de la même façon que des jours courts (Chemineau et al 1986, English et al 1986, Chemineau et al 1992).

La démonstration définitive de l'implication de la mélatonine a été faite par des expériences de remplacement chez des animaux pinéalectomisés (Bittman et al 1983a et 1984, Goldman et Nelson 1993). La perfusion de mélatonine dans la circulation périphérique pour mimer des profils de sécrétion de mélatonine de type « jours courts » ou « jours longs " peut reproduire les effets des jours courts ou des jours longs. Ainsi, des brebis pinéalectomisées, ovariectomisées et traitées avec un implant d'œstradiol, transférées de jours longs en jours courts sont dépourvues de mélatonine et ne montrent aucune modification de taux de LH. Si, au moment du transfert, elles reçoivent une perfusion quotidienne de mélatonine qui rétablit des niveaux élevés de mélatonine pendant 16 heures 
toutes les nuits (profil "jours courts»), une stimulation de la sécrétion de LH est observée après environ 50 jours, soit une réponse de type " jours courts " classique (Bittman et Karsch 1984).

\section{d / Paramètres critiques du rythme de sécrétion de mélatonine}

Le rythme circadien de sécrétion de mélatonine traduit les effets de la photopériode sur la fonction de reproduction. Cependant, il reste à déterminer quel est le paramètre de ce rythme qui est critique pour obtenir ces effets. Trois paramètres principaux ont été considérés : l'amplitude (différence entre niveaux nocturnes et diurnes), la phase (présence de mélatonine à un moment donné du nycthémère qui coïncide avec une période de sensibilité à cette hormone) ou la durée de sécrétion (durée quotidienne de présence de niveaux élevés). L'hypothèse " amplitude » n'a pas reçu beaucoup d'attention car l'amplitude du rythme n'est pas différente en jours longs et en jours courts. De plus, elle semble trop variable entre animaux pour constituer une caractéristique fiable du rythme capable de traduire l'information (Malpaux et al 1987).

Chez le hamster doré, l'injection de mélatonine en fin d'après-midi produit un effet " jours courts " (inhibition de l'activité reproductrice) alors qu'à d'autres moments, un tel effet n'est pas obtenu (Tamarkin et al 1976). Cette observation suggère qu'il existe un rythme de sensibilité à la mélatonine. Toutefois, l'absence de mesure des taux de mélatonine dans ces expériences ne permet pas d'exclure la possibilité que le pic de mélatonine exogène, ainsi administré peu de temps avant le début de la nuit, et la sécrétion endogène se fondent pour aboutir à un allongement de la durée de présence de mélatonine. Des expériences plus récentes rapportent le même type de différences de réponse en fonction du moment du nycthémère chez des animaux pinéalectomisés chez lesquels cette critique ne s'applique pas (Stetson et Watson-Whytmire 1986). Néanmoins, la plupart des résultats disponibles sont en faveur de l'hypothèse "durée ». En particulier, chez la brebis et le hamster sibérien, la perfusion de mélatonine produit toujours une réponse caractéristique de la durée de perfusion, indépendamment de la durée d'éclairement ou de la relation de phase entre le cycle lumineux et le moment de la perfusion (Carter et Goldman 1983, Karsch et al 1984, Wayne et al 1988). Des données en faveur de l'hypothèse "durée " ont également été obtenues chez le hamster doré, le vison et la souris à " pattes blanches " (Dowell et Lynch 1987, Bonnefond et al 1989, Goldman et Nelson 1993). Bien que la plupart des données disponibles soient en faveur de l'hypothèse "durée ", l'existence d'un rythme de sensibilité entraîné par la mélatonine elle-même a été peu explorée (Pitrosky 1994).

Que la durée ou la phase soit la caractéristique critique du rythme de sécrétion de mélatonine, elles ne sont pas absolues. En effet, il a été démontré dans plusieurs situations physiologiques que des profils de mélatonine caractérisés par une durée et une phase identiques pouvaient induire des réponses opposées. Par exemple, si des brebis ovariectomisées et traitées avec un implant d'œstradiol, exposées initialement à des jours longs (16L) sont soumises à 13L, une stimulation de la sécrétion de LH s'ensuit. A l'opposé, si des brebis sont initialement exposées à des jours courts (10L), leur exposition à $13 \mathrm{~L}$ se traduit par une inhibition de la sécrétion de LH (Robinson et Karsch 1987). Par conséquent, le même signal photopériodique (13L) induit des effets stimulants ou inhibiteurs selon la photopériode à laquelle les animaux ont été préalablement soumis. Les profils de sécrétion de mélatonine mesurés dans cette expérience indiquent que, dans toutes ces situations, la sécrétion de mélatonine dure pendant toute la nuit. Les animaux ont donc répondu différemment au même signal " mélatonine "selon la nature de leur exposition préalable à la mélatonine. A un moment donné, l'animal n'interprète donc pas une durée ou une phase du rythme de sécrétion de mélatonine dans l'absolu mais relativement à celles qu'il a perçues antérieurement.

\section{3 / Mode d'action de la mélatonine}

\section{a / Un effet majeur au niveau du système nerveux central}

La mélatonine peut agir à différents niveaux de l'axe hypothalamo-hypophysaire et gonadique. Toutefois, une étape clé de son action implique des événements au niveau du système nerveux central. En particulier, l'effet majeur de la mélatonine est de modifier la fréquence de libération du LHRH (luteinizing hormone releasing hormone, ou gonadolibérine) hypothalamique, ce qui, par voie de conséquence, change la fréquence de libération de la LH et l'activité des gonades. Ainsi, des brebis ovariectomisées, traitées avec un implant sous-cutané d'œstradiol et soumises à des jours longs, se caractérisent par une fréquence de libération de LHRH de l'ordre de 1 pulse par période de 6 heures. Le traitement de tels animaux avec un implant sous-cutané de mélatonine qui produit un effet "jours courts ", va se traduire par une stimulation de la libération pulsatile de LHRH pour atteindre une fréquence de l'ordre de 10 pulses/6 heures (figure 6 ; Viguié et al 1995a). Il est à noter que l'intervalle de 40 à 60 jours observé entre le début d'un traitement par des jours courts et la stimulation de la sécrétion de LH ou de l'ovulation chez la brebis se retrouve entre le début du traitement par la mélatonine et la stimulation de la sécrétion de LHRH, ce qui démontre que les mécanismes responsables de ce délai de latence sont essentiellement d'origine nerveuse. 
Figure 6. Modification de la sécrétion pulsatile de $L H R H$ et de $L H$ par la mélatonine chez la brebis lle-deFrance. Le traitement de brebis, ovariectomisées et traitées avec un implant sous-cutané d'œstradiol, par un implant de mélatonine induit après 40 à 60 jours une augmentation de la fréquence de libération de LHRH et de LH et, en conséquence, des taux plasmatiques moyens de LH (adapté de Viguié et al 1995a).

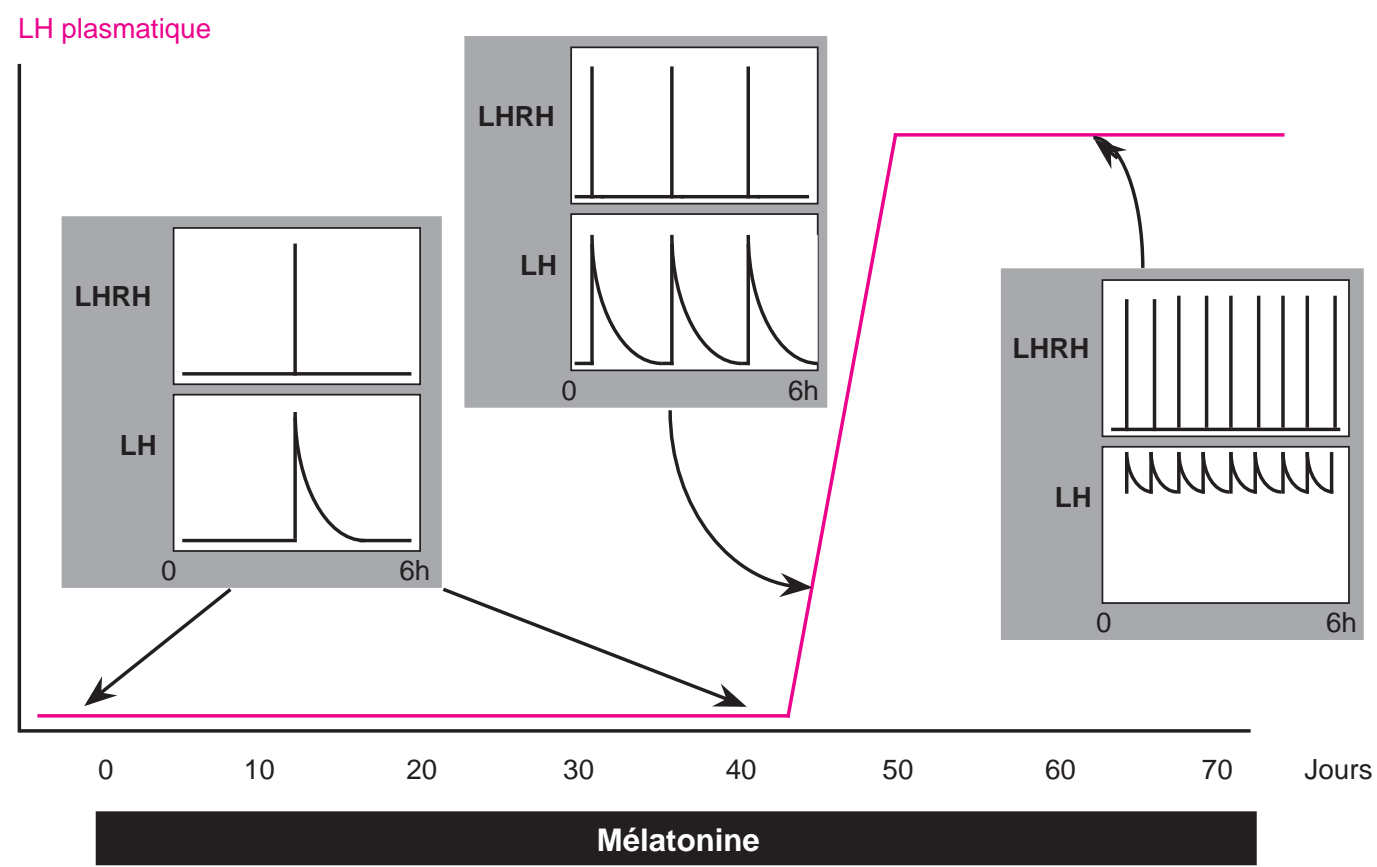

\section{b / Sites d'action de la mélatonine}

Des récepteurs à la mélatonine ont été mis en évidence dans de nombreuses structures. Toutefois, dans toutes les espèces de mammifères étudiées, la plus forte densité de récepteurs à la mélatonine est trouvée dans la pars tuberalis de l'hypophyse (PT, figure $3 \mathrm{~b}$; de Reviers et al 1989, Bittman 1993). Il existe même deux espèces très sensibles à la photopériode, le vison et le furet, chez lesquelles la pars tuberalis est le seul site où une liaison de la mélatonine a été détectée (BoissinAgasse et al 1992, Bittman 1993). Ces observations ont conduit à proposer la pars tuberalis comme le site principal d'action de la mélatonine. Cependant, des microimplants de mélatonine disposés contre la pars tuberalis ou à l'intérieur de celle-ci sont incapables de stimuler la sécrétion de LH chez des brebis ovariectomisées et traitées avec un implant d'œstradiol (Malpaux et al 1994 et 1995). Ces microimplants étant capables de stimuler la sécrétion de LH lorsqu'ils sont disposés dans le troisième ventricule, cette observation suggère que la pars tuberalis n'est pas le site d'action de la mélatonine pour ses effets sur la fonction de reproduction.

La pose de microimplants de mélatonine dans divers sites hypothalamiques a permis d'identifier des sites potentiels d'action. En effet, des microimplants de mélatonine disposés dans l'hypothalamus médiobasal permettent de stimuler la sécrétion de $\mathrm{LH}$ chez la brebis ou l'activité testiculaire chez le bélier de la même manière que des jours courts ou des implants sous-cutanés (figure 3b ; Lincoln et Maeda 1992, Malpaux et al 1993). Lorsque ces microimplants sont disposés dans l'hypothalamus antérieur ou dorsolatéral ou dans l'aire pré-optique, ils n'ont aucun effet détectable sur la sécrétion de LH (Malpaux et al 1993). Ces résultats suggèrent une localisation des sites d'action de la mélatonine dans l'hypothalamus médiobasal où des récepteurs à la mélatonine en faible densité ont été identifiés (Bittman et Weaver 1990, Chabot et al 1994). Cette hypothèse est renforcée par l'observation des effets de la lésion de l'hypothalamus médiobasal chez le hamster doré. En effet, chez de tels animaux, après lésion, la mélatonine n'a plus d'effet sur l'activité de reproduction (Maywood et Hastings 1995). Le clonage récent d'un récepteur membranaire à la mélatonine et la mise en évidence d'un récepteur nucléaire qui est capable de lier la mélatonine pourraient permettre de résoudre l'incohérence apparente entre la localisation des sites d'action et de liaison (Reppert et al 1994, Becker-André et al 1994).

\section{c / Médiateurs de l'action de la mélatonine sur les neurones à LHRH}

$\mathrm{Au}$ niveau du système nerveux central, le point final de l'action de la mélatonine est la modification de la sécrétion pulsatile des neurones à LHRH. Les corps cellulaires des neurones à LHRH sont localisés en majorité $(60$ $\%$ ) dans l'aire pré-optique (figure $3 \mathrm{a}$ et $\mathrm{b}$; Caldani et al 1988). Ces neurones se projettent dans l'éminence médiane pour libérer le LHRH dans le système porte hypothalamohypophysaire (figure $3 a$ et b). L'absence de récepteurs à la mélatonine et d'action de

\section{L'effet majeur de la mélatonine est de modifier la fréquence de libération du LHRH et donc la fréquence de libération de la LH et l'activité des gonades.}


microimplants de mélatonine dans la région septo-préoptique suggère que l'action de la mélatonine sur les neurones à $\mathrm{LHRH}$ est indirecte et met en jeu des interneurones. Cette hypothèse est renforcée par le long délai entre le début de l'action de la mélatonine et la modification de la sécrétion de LHRH (40 à 60 jours, figure 6 ; Viguié et al 1995a). L'implication de différents types de neurones et de neuromédiateurs est suspectée ainsi qu'une interaction de ces neurones avec les hormones thyroïdiennes.

\section{- Dopamine}

De nombreux résultats suggèrent que la dopamine (DA) exerce une action inhibitrice sur la sécrétion de LH et que l'intensité de cette action peut être modulée par l'action de la photopériode (Thiéry et al 1995). Ainsi, chez la brebis entière ou ovariectomisée et traitée avec un implant d'œstradiol, l'administration systémique d'un antagoniste dopaminergique (pimozide) permet de stimuler la sécrétion de LH (Meyer et Goodman 1985, Le Corre et Chemineau 1993). A l'opposé, l'administration intracérébroventriculaire d'un agoniste dopaminergique pendant la saison sexuelle inhibe la sécrétion de LH (Curlewis et al 1990). De plus, la lésion neurotoxique d'un noyau dopaminergique hypothalamique (A15) induit une augmentation de la fréquence de libération de la LH pendant la saison d'anœstrus chez la brebis (Thiéry et al 1989). Dans ce noyau, la synthèse et la libération de dopamine sont stimulées par l'œstradiol chez des brebis exposées à des jours longs, ce qui suggère que cette structure pourrait être impliquée dans les variations de rétroaction négative de l'œstradiol (Gayrard et al 1992, 1994).

L'éminence médiane semble être un site particulièrement important où la dopamine est mise en jeu dans l'action de la mélatonine. En effet, dans ce site, l'exposition à des jours courts ou le traitement par un implant de mélatonine induit une diminution de la concentration de dopamine (Thiéry 1991) et de l'activité de synthèse de la dopamine (Viguié et al 1994, Viguié 1995). Par ailleurs, le blocage de la synthèse de dopamine par perfusion locale d'un inhibiteur induit une stimulation de la sécrétion de LH chez des brebis exposées à des jours longs (Viguié 1995). Ces résultats suggèrent qu'une modulation de l'activité dopaminergique au niveau de l'éminence médiane constitue un des mécanismes responsables de l'action de la mélatonine sur la sécrétion de LHRH chez la brebis.

\section{- Noradrénaline}

La noradrénaline semble exercer une inhibition de la sécrétion pulsatile de LH pendant la saison d'anœstrus. En effet, l'injection par voie intraveineuse d'un antagoniste noradrénergique (phénoxybenzamine) provoque une augmentation de la sécrétion de LH (Meyer et Goodman 1985). Par ailleurs, l'œstradiol augmente les concentrations intra et extra- cellulaires d'un métabolite de la noradrénaline (MHPG) du noyau A15 pendant les jours longs (Thiéry 1991), suggérant que la noradrénaline pourrait être impliquée dans l'établissement du rétrocontrôle négatif de la sécrétion de LH par l'œstradiol lors de l'exposition à des jours longs.

\section{- Sérotonine}

La sérotonine semble aussi exercer une inhibition de la sécrétion de LH pendant la saison d'anœstrus. En effet, l'injection par voie intraveineuse d'un antagoniste (cyproheptadine) de cette indolamine provoque une stimulation de la sécrétion de LH pendant la saison d'anœstrus chez des brebis ovariectomisées (Meyer et Goodman 1986). La sérotonine pourrait jouer un rôle inhibiteur essentiel pendant l'état réfractaire aux jours courts. Chez des brebis entières, l'inhibition de la synthèse de sérotonine provoque un retard dans l'apparition de l'état réfractaire aux jours courts (Chemineau et al 1991). L'injection de cyproheptadine à des brebis ovariectomisées, traitées avec un implant d'œstradiol et réfractaires aux jours courts provoque une stimulation de la sécrétion de LH (Le Corre et Chemineau 1993). Enfin, la densité de liaison de kétansérine tritiée (un ligand des récepteurs sérotoninergiques) est plus élevée dans la partie ventrolatérale de l'hypothalamus chez des brebis ovariectomisées et traitées avec un implant d'œstradiol exprimant une réponse "jours courts " (sécrétion de LH élevée) que chez des animaux réfractaires à cette photopériode (sécrétion de LH faible ; Le Corre et al 1994).

\section{- Acides aminés excitateurs}

Chez le hamster et le mouton, l'injection de NMDA (N-méthyl,D,L,aspartate, un agoniste des acides aminés excitateurs tels que les acides aspartique et glutamique) cause une libération massive de LH quand l'activité gonadotrope de ces animaux est inhibée par la photopériode (Lincoln et Wu 1991, Hui et al 1992, Urbanski 1992, Viguié et al 1995b). A l'opposé, la libération de LH est faible ou inexistante quand le NMDA est injecté sur des animaux présentant une activité gonadotrope élevée. Ces variations de libération de LH en réponse au NMDA en fonction de l'état physiologique traduisent des changements identiques de libération de LHRH (Viguié et al 1995b). Cette variation de la libération de LHRH induite par le NMDA peut simplement traduire un changement dans les stocks immédiatement libérables de LHRH ; en effet, ces stocks sont plus importants chez des animaux à faible activité gonadotrope. Alternativement, elle pourrait traduire un changement dans la stimulation endogène des récepteurs au NMDA qui ferait partie des mécanismes responsables de l'action de la mélatonine. L'observation d'une variation de la densité des récepteurs au NMDA en fonction de la photopériode chez le hamster est en faveur de cette seconde possibilité (Urbanski et Pierce 1992). 


\section{- Hormones thyroüdiennes}

Des brebis ou des béliers thyroïdectomisés ne montrent plus de variations saisonnières d'activité sexuelle ou de sécrétion de LH (Nicholls et al 1988, Mœnter et al 1991, Parkinson et Follett 1994). L'administration de T4 annule les effets de la thyroïdectomie en rétablissant l'inhibition de la sécrétion de $\mathrm{LH}$ au moment normal de la fin de la saison sexuelle (Webster et al 1991). Les hormones thyroïdiennes sont impliquées dans des phénomènes de neuroplasticité. Le rôle des hormones thyroïdiennes dans la régulation saisonnière de la reproduction pourrait-il être de permettre des changements structuraux neuronaux que la mélatonine aurait pour fonction d'induire? Un tel rôle de la mélatonine a été suggéré par plusieurs observations. La densité synaptique des afférences sur les neurones à LHRH dans l'aire préoptique est deux fois plus importante pendant la saison sexuelle (Karsch et al 1987). Par ailleurs, des changements de densité de récepteurs sérotoninergiques sont observés entre différentes situations photopériodiques (Le Corre et al 1994).

\section{Conclusion}

La photopériode influence l'activité de reproduction par des mécanismes complexes et variés qui relient l'œil aux gonades. L'information lumineuse est successivement traitée par voie nerveuse (liaison œil-glande pinéale), endocrinienne (mélatonine), de nouveau nerveuse (liaison cible de la mélatonine-neurones à LHRH) et enfin de nouveau endocrinienne (LHRH et gonadotropines). De plus, l'action de la photopériode sur l'activité de reproduction est un modèle d'interaction entre des rythmes endogènes de périodes différentes : un rythme circadien de sécrétion de mélatonine qui permet de synchroniser un rythme circannuel de reproduction dont les différentes phases se caractérisent par des fréquences variables de libération de LHRH. Une étape importante et particulièrement mal connue de cette régulation concerne les mécanismes impliqués dans l'action de la mélatonine sur la libération de LHRH. Une meilleure connaissance de ces mécanismes devrait permettre d'appréhender la nature du rythme endogène circannuel de reproduction qui est l'élément clé de la saisonnalité de la reproduction.

\section{Références bibliographiques}

Arendt J., 1986. Role of the pineal gland and melatonin in seasonal reproductive functions in mammals. Oxford Rev. Reprod. Biol., 8, 266-320.

Becker-André M., Wiesenberg I., Schaeren-Wiemers N., André E., Missbach M., Saurat J.H., Carlberg C., 1994. Pineal gland hormone melatonin binds and activates an orphan of the nuclear receptor superfamily. J. Biol. Chem., 269, 28531-28534.

Beltrán de Heredia I., Daveau A., Chemineau P., 1993. Stability in the amplitude and duration of the nycthemeral melatonin rhythm after photoperiodic changes in the Ile-de-France ewe. 6th Colloquium of the European Pineal Society, 23-27 Juillet 1993, Copenhagen, abst C3.

Bittman E.L., 1993. The sites and consequences of melatonin binding in mammals. Amer. Zool., 33, 200-211.

Bittman E.L., Karsch F.J., 1984. Nightly duration of pineal melatonin secretion determines the reproductive response to inhibitory day lengths in the ewe. Biol. Reprod., 30, 585-593.

Bittman E.L., Weaver D., 1990. The distribution of melatonin binding sites in neurœndocrine tissues of the ewe. Biol. Reprod., 43, 986-993.

Bittman E.L., Dempsey R.J., Karsch F.J., 1983a. Pineal melatonin secretion drives the reproductive response to daylength in the ewe. Endocrinology, $113,2276-83$

Bittman E.L., Karsch F.J., Hopkins J.W., 1983b. Role of the pineal gland in ovine photoperiodism : regulation of seasonal breeding and the negative feedback effects of estradiol upon luteinizing hormone secretion. Endocrinology, 113, 329-336.
Boissin-Agasse L., Barberis C., Audigier S., Roch G., Boissin J., 1992. Localization of melatonin binding sites in the pars tuberalis of the mink at three times during the seasonal testicular cycle. Neurosci. Lett., $144,147-151$.

Bonnefond C., Walker A..P., Stutz J.A., Maywood E., Juss T.S., Herbert J., Hastings M.H., 1989. The hypothalamus and photoperiodic control of FSH secretion in the male Syrian hamster. J. Endocr. $122,247-254$.

Broadway J., Arendt J., Folkard S., 1987. Bright light phase shifts the human melatonin rhythm during the antarctic winter. Neurosci. Lett., 79, 185189.

Caldani M., Batailler M., Thiéry J.C., Dubois M.P., 1988. LH-RH immunoreactive structures in the sheep brain. Histochem., 89, 129-139.

Carter D.S., Goldman B.D., 1983. Antigonadal effects of timed melatonin infusion in pinealectomized male Djungurian hamsters (Phodopus sungorus sungorus) : duration is the critical parameter. Endocrinology, 113, 1261-1267.

Chabot V., Caldani M., de Reviers M.M., Pelletier J., 1994. Localisation and quantification of melatonin receptors in the diencephalon and posterior telencephalon of the sheep brain. XXIII ${ }^{\mathrm{e}}$ Colloque de la Société de Neurœndocrinologie Expérimentale, Sophia Antipolis, 1-3 Septembre 1994.

Chemineau P., Normant E., Ravault J.P., Thimonier J., 1986. Induction and persistence of pituitary and ovarian activity in the out-of-season lactating dairy goat after a treatment combining a skeleton photoperiod, melatonin and the male effect. J. Reprod. Fert., 78, 497-504. 
Chemineau P., Martin G.B., Saumande J., Normant E., 1988. Seasonal and hormonal control of pulsatile LH secretion in the dairy goat (Capra hircus). J. Reprod. Fert., 83, 91-98.

Chemineau P., Daveau A., Maurice F., 1991. L'utilisation de PCPA, un inhibiteur de la synthèse de sérotonine, retarde l'apparition de l'état réfractaire aux jours courts chez la brebis Ile-de-France. 4ème Colloque Biotechnocentre, Seillac (France), 30-31 Octobre, abst 42.

Chemineau P., Malpaux B., Delgadillo J.A., Guérin Y., Ravault J.P., Thimonier J., Pelletier J., 1992. Control of sheep and goat reproduction : Use of light and melatonin. Anim. Reprod. Sci., 30, 157-184.

Curlewis J.D., Naylor A.M., McNeilly A.S., 1990. Dopamine D-1 and D-2 receptor regulation of $\mathrm{LH}$ and prolactin secretion in the ewe. Neurœndocrino$\operatorname{logy} 52$, sup. 1,92 .

Dacheux J.L., Pisselet C., Blanc M.R., Hochereaude-Reviers M.T., Courot M., 1981. Seasonal variations in rete testis fluid secretion and sperm production in different breeds of ram. J. Reprod. Fert., 61, 363-371.

Delgadillo J.A., Chemineau P., 1992. Abolition of the seasonal release of luteinizing hormone and testosterone in Alpine male goats by short photoperiodic cycles. J. Reprod. Fert., 94, 45-55.

de Reviers M.-M., Ravault J.-P., Tillet Y., Pelletier J., 1989. Melatonin binding sites in the sheep pars tuberalis. Neurosci. Lett., 100, 89-93.

Domanski E., Przekop F., Polkowska J., 1980. Hypothalamic centres involved in the control of gonadotropins secretions. J. Reprod. Fert., 58, 493-499.

Dowell S.F., Lynch G.R., 1987. Duration of the melatonin pulse in the hypothalamus controls testicular function in pinealectomized mice (Peromyscus Leucopus). Biol. Reprod., 36, 1095-1101.

Ducker M.J., Bowman J.C., Temple A., 1973. The effect of constant photoperiod on the expression of œstrus in the ewe. J. Reprod. Fert. Suppl., 19, 143150.

Ebling F.J.P., Lincoln G.A., Wollnik F., Anderson N., 1988. Effects of constant darkness and constant light on circadian organization and reproductive responses in the ram. J. Biol. Rhythms, 3, 365-384.

English J., Poulton A.L., Arendt J., Symons A.M., 1986. A comparison of the efficiency of melatonin treatments in advancing œstrus in ewes. J. Reprod. Fert., 77, 321-27.

Gayrard V., Malpaux B., Thiéry J.C., 1992. Oestradiol increases the extracellular levels of amine metabolites in the ewe hypothalamus during anœstrus : a microdialysis study. J. Endocr., 135, 421430 .

Gayrard V., Malpaux B., Tillet Y., Thiéry J.C., 1994. Estradiol increases tyrosine hydroxylase activity of the A15 nucleus dopaminergic neurons during long days in the ewe. Biol. Reprod., 50, 1168-1177.

Goldman B.D., Nelson R.J., 1993. Melatonin and seasonality in mammals. In : Yu H.S., Reiter R.J. (Eds), Melatonin : Biosynthesis, Physiological Effects, and Clinical Applications, 225-252. CRC Press, Boca Raton, Florida.

Gwinner E., 1986. Circannual rhythms. Berlin, Springer-Verlag, $154 \mathrm{pp}$.
Herbert J., Stacey P.M., Thorpe D.H., 1978. Recurrent breeding seasons in pinealectomized or opticnerved-sectioned ferrets. J. Endocr., 78, 389-397.

Hirata F., Hayaishi O., Tokuyama, T., Senoh, S., 1974. In vitro and in vivo formation of two new metabolites of melatonin. J. Biol. Chem., 249, 13111313

Hoffman K., 1981. Photoperiodic function of the mammalian pineal gland. In : Oksche A., Pévet P. (eds), The pineal organ : Photobiology-biochronology-endocrinology, 123-138. Elsevier, Amsterdam.

Howles C.M., Craigon J., Haynes N.B., 1982. Long term rhythms of testicular volume and plasma prolactin concentrations in rams reared for 3 years in constant photoperiod. J. Reprod. Fert., 65, 439-446.

Hui Y., Hastings M.H., Maywood E.S., Ebling F.J.P., 1992. Photoperiodic regulation of glutamatergic stimulation of secretion of luteinizing hormone in male Syrian hamster. J. Reprod. Fert., 95, 935-946.

Kanematsu N, Mori Y., Hayashi S., Hoshino, K., 1989. Presence of a distinct 24-hour melatonin rhythm in the ventricular cerebrospinal fluid of the goat. J. Pineal Res., 7, 143-152.

Karsch F.J., Bittman E.L., Foster D.L., Goodman R.L., Legan S.J., Robinson J.E., 1984. Neurœndocrine basis of seasonal reproduction. Recent Prog. Horm. Res., 40, 185-232.

Karsch F.J., Lehman M.N., Silverman A.J., 1987. Seasonal plasticity in synaptic input to luteinizing hormone-releasing hormone (LHRH) neurons in the sheep. Neurosci. Abstr., 13, 1527.

Karsch F.J., Robinson J.E., Woodfill C.J.I., Brown M.B., 1989. Circannual cycles of luteinizing hormone and prolactin secretion in ewes during a prolonged exposure to a fixed photoperiod : evidence for an endogenous reproductive rhythm. Biol. Reprod., $41,1034-1046$

Klein D.C., 1985. Photoneural regulation of the mammalian pineal gland. CIBA Foundation Symposia, $117,38-56$.

Klein D.C., Smoot R., Weller J.L., Higa S., Markley S.P., Creed G.J., Jacobowitz D.M., 1983. Lesions of the paraventricular nucleus area of the hypothalamus disrupt the suprachiasmatic to spinal cord circuit in the melatonin rhythm generating system. Brain Res., 10, 647-652.

Legan S.J., Karsch F.J., 1983. Importance of retinal photoreceptors to the photoperiodic control of seasonal breeding in the ewe. Biol. Reprod., 29, 316-325.

Legan S.J., Winans S.S., 1981. The photoneurondocrine control of seasonal breeding in the ewe. Gen. Comp. Endoc., 45, 317-328.

Le Corre S., Chemineau P., 1993. Control of photoperiodic inhibition of luteinizing hormone secretion by dopaminergic and serotoninergic systems in ovariectomized Ile-de-France ewes supplemented with œstradiol. J. Reprod. Fert., 97, 367-373.

Le Corre S., Segu L., Caldani M., Chemineau P., 1994. Differences in ketanserin binding in the ventromedial hypothalamus of ewes responsive or refractory to short days. Neurœndocrinology, 60, 589-600.

Lincoln G. A., 1979. Photoperiodic control of seasonal breeding in the ram : participation of the cranial sympathetic nervous system. J. Endocr., 82,135-147. 
Lincoln G.A., Maeda K.I., 1992. Reproductive effects of placing microimplants of melatonin in the mediobasal hypothalamus and preoptic area in rams. J. Endocrinol., 132, 201-215.

Lincoln G.A., Wu F.C.W., 1991. Luteinizing hormone responses to N-methyl-D,L-Aspartate during a photoperiodically-induced reproductive cycle in the ram. J. Neurœndocrinol., 3, 309-317.

Malpaux B., Karsch F.J., 1990. A role for short days in sustaining seasonal reproductive activity in the ewe. J. Reprod. Fert., 90, 555-562.

Malpaux B., Robinson J.E., Brown M.B., Karsch F.J., 1987. Reproductive refractoriness of the ewe to inductive photoperiod is not caused by inappropriate secretion of melatonin. Biol. Reprod., 36, 1333-1341.

Malpaux B., Mœnter S.M., Wayne N.L., Woodfill C.J.I., Karsch F.J., 1988a. Reproductive refractoriness of the ewe to inhibitory photoperiod is not caused by alteration of the circadian secretion of melatonin. Neuroendocrinology, 48, 264-270.

Malpaux B., Robinson J.E., Brown M.B., Karsch F.J., 1988b. Importance of changing photoperiod and melatonin secretory pattern in determining the length of the breeding season in the Suffolk ewe. J. Reprod. Fert., 83, 461-471.

Malpaux B., Wayne N.L., Karsch F.J., 1988c. Termination of the breeding season in the Suffolk ewe : involvement of an endogenous rhythm of reproduction. Biol. Reprod., 39, 254-263.

Malpaux B., Robinson J.E., Wayne N.L., Karsch F.J., 1989. Regulation of the onset of the breeding season of the ewe : importance of longs days and of an endogenous reproductive rhythm. J. Endocr., $122,269-278$.

Malpaux B., Daveau A., Maurice F., Gayrard V., Thiéry J.C., 1993. Short-day effects of melatonin on luteinizing-hormone secretion in the ewe : Evidence for central sites of action in the mediobasal hypothalamus. Biol. Reprod., 48, 752-760.

Malpaux B., Daveau A., Maurice F., Locatelli A., Thiéry J.C., 1994. Evidence that melatonin binding sites in the pars tuberalis do not mediate the photoperiodic actions of melatonin on $\mathrm{LH}$ and prolactin secretion in ewes. J. Reprod. Fert., 101, 625-632.

Malpaux B., Skinner D.C., Maurice F., 1995. The ovine pars tuberalis dœs not appear to be targeted by melatonin to modulate luteinizing hormone secretion, but may be important for prolactin release. J. Neuroendocrinol., 7, 199-206.

Martinet L., Mondain-Monval M., 1991. Rythmes de reproduction et facteurs de l'environnement. In : Thibault C., Levasseur M.C. (Eds), La reproduction chez les mammifères et l'homme, 589-610. Coédition Ellipses-INRA, Paris.

Mauléon P., Rougeot J., 1962. Régulation des saisons sexuelles chez des brebis de races différentes au moyen de divers rythmes lumineux. Ann. Biol. anim. Bioch. Biophys., 2, 209-222.

Maywood E.S., Hastings M.H., 1995. Lesions of iodomelatonin-binding sites of the mediobasal hypothalamus spare the lactotropic, but block the gonadotropic response of male Syrian hamsters to short photoperiod and to melatonin. Endocrinology, 136, $144-153$.
Meyer S.L., Goodman R.L., 1985. Neurotransmitters involved in mediating the steroid-dependent suppression of pulsatile luteinizing hormone secretion in anestrous ewes : effects of receptor antagonists. Endocrinology, 116, 2054-2061.

Meyer S.L., Goodman R.L., 1986. Separate neural systems mediate the steroid-dependent and steroidindependent suppression of tonic luteinizing hormone secretion in the anœstrus ewe. Biol. Reprod., $35,562-571$.

Mœnter S.M., Woodfill C.J.I., Karsch F.J., 1991. Role of the thyroid gland in seasonal reproduction : thyroidectomy blocks seasonal suppression of reproductive neurœendocrine activity in ewes. Endocrinology, 128, 1337-1344.

Montgomery G.W., Martin G.B., Pelletier J., 1985. Changes in pulsatile LH secretion after ovariectomy in Ile-de-France ewes in two seasons. J. Reprod. Fert., 73, 173-183.

Nicholls T.J., Follett B.K., Goldsmith A.R., Pearson H., 1988. Possible homologies between photorefractoriness in sheep and birds : the effect of thyroidectomy on the length of the ewe's breeding season. Reprod. Nutr. Develop., 28, 375-385.

Ortavant R., Pelletier J., Ravault J.P., Thimonier J., Volland-Nail P., 1985. Photoperiod : main proximal and distal factor of the circannual cycle of reproduction in farm animals. Oxford Rev. Reprod. Biol., 7, 305-345.

Paterson A.M., Foldes A., 1994. Melatonin and farm animals : endogenous rhythms and exogenous applications. J. Pineal Res., 16, 167-177.

Parkinson T.J., Follett B.K., 1994. Effect of thyroidectomy upon seasonality in rams. J. Reprod. Fert., $101,51-58$

Pelletier J., Ortavant R., 1975. Photoperiodic control of LH release in the ram : I light-androgens interaction. Acta Endocr. Copenh., 78, 442-450.

Pitrosky B., 1994. Mélatonine et photopériodisme : importance des différents paramètres du profil de sécrétion de la mélatonine chez le hamster syrien. Thèse Université Strasbourg, 148 pp.

Reiter R.J., 1981. The mammalian pineal gland : structure and function. Am. J. Anat., 162, 287-313.

Reppert S.M., Weaver D.R., Ebisawa T., 1994. Cloning and characterization of a mammalian melatonin receptor that mediates reproductive and circadian responses. Neuron, 13, 1177-1185.

Robinson J.E., Karsch F.J., 1984. Refractoriness to inductive day lengths terminates the breeding season of the Suffolk ewe. Biol. Reprod., 31, 656-663.

Robinson J.E., Karsch F.J., 1987. Photoperiodic history and a changing melatonin pattern can determine the neurondocrine response of the ewe to day length. J. Reprod. Fert., 80,159-165.

Robinson J.E., Radford H.M., Karsch F.J., 1985a. Seasonal changes in pulsatile luteinizing hormone (LH) secretion in the ewe, relationship of frequency of $\mathrm{LH}$ pulses to day length and response to estradiol negative feedback. Biol. Reprod., 33, 324-334.

Robinson J.E., Wayne N.L., Karsch F.J., 1985b. Refractoriness to inhibitory daylength initiates the breeding season of the Suffolk ewe. Biol. Reprod., $32,1024-1030$ 
Rollag M.D., Niswender G.D., 1976. Radioimmunoassay of serum concentrations of melatonin in sheep exposed to different lighting regimens. Endocrinology, 98, 482-489.

Shaw P.F., Kennaway D.J., Seamark R.F., 1989. Evidence of high concentrations of melatonin in lateral ventricular cerebrospinal fluid of sheep. J. Pineal Res., 6, 201-208.

Stetson M.H., Watson-Whytmire M., 1986. Effects of exogenous and endogenous melatonin on gonadal function in hamsters. Biol. Reprod., 34, 664-669.

Sugden D., 1989. Melatonin biosynthesis in the mammalian pineal gland. Experientia, 45, 922-932.

Swanson L.H, Kuypers H.G.J.M., 1980. The paraventricular nucleus of the hypothalamus : cytoarchitectonic subdivisions and organization of projections to the pituitary, dorsal vagal complex, and spinal cord as demonstrated by retrograde fluorescence double-labelling methods. J. Comp. Neurol., 194, 550-570.

Tamarkin L., Westrom W.K., Hamill A.I., Goldman B.D., 1976. Effect of melatonin on the reproductive systems of male and female Syrian hamsters : diurnal rhythm in sensitivity to melatonin. Endocrinology, 99, 1534-1541.

Tessonneaud A., Locatelli A., Caldani M., ViguierMartinez M.C., 1995. Bilateral lesions of the suprachiasmatic nuclei alter the nocturnal melatonin secretion in sheep. J. Neurœndocrinol., 7, 145-152.

Thiéry J.C., 1991. Monoamine content of the stalkmedian eminence and hypothalamus in adult female sheep as affected by daylength. J. Neurœndocrinol., $3,407-411$.

Thiéry J.C., Martin G.B., Tillet Y., Caldani M., Quentin M., Jamain C., Ravault J.P., 1989. Role of hypothalamic catecholamines in the regulation of luteinizing hormone and prolactin secretion in the ewe during seasonal anœstrus. Neurœndocrinology, $49,80-87$.

Thiéry J.C., Gayrard V., Le Corre S., Viguié C., Martin G.B., Chemineau P., Malpaux B., 1995. Dopaminergic control of LH secretion by the A15 nucleus in anœstrous ewes. J. Reprod. Fert., Suppl. 49, 285296.

Thimonier J., 1989. Contrôle photopériodique de l'activité ovulatoire chez la brebis. Existence de rythmes endogènes, Thèse Université François Rabelais, Tours, $112 \mathrm{pp}$.

Thimonier J., Mauléon P., 1969. Variations saisonnières du comportement d'œstrus et des activités ovarienne et hypophysaire chez les ovins. Ann. Biol. anim. Bioch. Biophys., 9, 223-250.

Thimonier J., Sempéré A., 1989. La reproduction chez les cervidés. INRA Prod. Anim., 2, 5-21.

Thimonier J., Ravault J.P., Ortavant R., 1978. Plasma prolactin variations and cyclic ovarian activity in ewes submitted to different light regimens. Ann Biol. anim. Bioch. Biophys., 18, 1229-1235.

Thwaites C.J., 1965. Photoperiodic control of breeding activity in the Southdown ewe with particular reference to the effects of an equatorial light regime. J. Agric. Sci. Camb., 65, 57-64

Urbanski H.F., 1992. Photoperiodic modulation of luteinizing hormone secretion in orchidectomized Syrian hamsters and the influence of excitatory amino acids. Endocrinology, 131, 1665-1669.
Urbanski H.K., Pierce M., 1992. Photoperiodic control of seasonal breeding in Syrian hamsters : involvement of excitatory amino acid receptors. Neurondocrinol. Lett., 14, 33-37.

Van Camp G., Ravault J.-P., Falcon J., Collin J.-P., Voisin P., 1991. Regulation of melatonin release and $\mathrm{N}$-acetyltransferase activity in ovine pineal cells. J. Neurœndocr., 3, 477-481.

Viguié C., 1995. Régulation de la sécrétion pulsatile de LHRH par la photopériode et la mélatonine chez la brebis : mise en évidence et caractérisation d'une étape dopaminergique. Thèse Université Montpellier II, $152 \mathrm{pp}$.

Viguié C., Thibault J., Thiéry J.C., Tillet Y., Malpaux B., 1994. Reduction of tyrosine hydroxylase activity in the stalk-median eminence of the ewe by short days in relation to changes in luteinizing hormone and prolactin secretion. Biol. Reprod., 50, Suppl. 1.

Viguié C., Caraty A., Locatelli A., Malpaux B., 1995a. Regulation of LHRH secretion by melatonin in the ewe. I Simultaneous delayed increase in LHRH and LH pulsatile secretion. Biol. Reprod., 52, $1114-1120$.

Viguié C., Caraty A., Locatelli A., Malpaux B., 1995b. Regulation of LHRH secretion by melatonin in the ewe. II Changes in N-Methyl-D,L-aspartic acid-induced LHRH release during the stimulation of LH secretion by melatonin. Biol. Reprod., 52, 1156-1161.

Wayne N.L., Malpaux B., Karsch F.J., 1988. How dœs melatonin code for day length in the ewe : Duration of nocturnal melatonin release or coincidence of melatonin with a light-entrained sensitive period ? Biol. Reprod., 39, 66-75.

Wayne N.L., Malpaux B., Karsch F.J., 1990. Photoperiodic requirements for timing the onset and duration of the breeding season of the ewe : Synchronization of an endogenous rhythm of reproduction. J. Comp. Physiol., A 166, 835-842.

Webster J.R., Mœnter S.M., Woodfill C.J.I., Karsch F.J., 1991. Role of the thyroid gland in seasonal reproduction. II. Thyroxine allows a season-specific suppression of gonadotropin secretion in sheep. Endocrinology, 129, 176-183.

Woodfill C.J.I., Robinson J.E., Malpaux B., Karsch F.J., 1991. Synchronization of the circannual reproductive rhythm of the ewe by discrete photoperiodic signals. Biol. Reprod., 45, 110-121.

Worthy K., Haresign W., 1983. Evidence that the onset of seasonal anœstrus in the ewe may be independent of increasing prolactin concentrations and daylength. J. Reprod. Fert., 69, 41-48.

Worthy K., Haresign W., Dodson S., McLeod B.J., Foxcroft G.R., Haynes N.B., 1985. Evidence that the onset of the breeding season in the ewe may be independent of decreasing plasma prolactin concentrations. J. Reprod. Fert., 75, 237-246.

Yeates N.T.M., 1949. The breeding season of the sheep with particular reference to its modification by artificial light. J. Agric. Sci. Camb, 39, 1-43.

Yu H.S., Tsin A.T.C., Reiter R.J., 1993. Melatonin : History, Biosynthesis, and Assay Methodology. In : Yu H.S., Reiter R.J. (Eds), Melatonin : Biosynthesis, Physiological Effects, and Clinical Applications, 116. CRC Press, Boca Raton, Florida. 


\begin{abstract}
Photoperiodic control of breeding activity.

Annual variations in daylength or photoperiod are responsible for an alternation between a breeding and a rest season in most animal species. Depending on its length, the photoperiod can stimulate or inhibit reproductive activity. Animals deprived of photoperiodic information, however, exhibit an endogenous rhythm of reproduction and the main role of photoperiod under natural conditions appears to be a synchronisation of this internal rhythm.

tion. This hormone is only secreted at night. Melatonin acts on the central nervous system to control the pulsatile secretion of LHRH. Its action on LHRH neurons is not direct ; it involves the use of interneurons. In addition, the melatonin-induced changes in LHRH secretion are seen after a long latency relative to the time of the change in the rhythm of melatonin secretion, 40 to 60 days in the ewe. The changes in LHRH secretion cause modifications in gonadotropic hormone secretion which in turn control the activity of the gonads.
\end{abstract}

Light information is perceived by the retina and, after neural processing, it is transformed into an endocrine signal, the rhythm of melatonin secre-
MALPAUX B., VIGUIÉ C., THIÉRY J.C., CHEMINEAU P., 1995. Contrôle photopériodique de la reproduction. INRA Prod. Anim., 9 (1), 9-23. 